A Platinum Open Access Journal for Organic Chemistry
Paper

Arkivoc 2022, part ii, 22-29

Free to Authors and Readers

DOAJ Seal

\title{
Synthesis of pyrazolo[1,5-a]pyrimidine ring as a possible bioisosteric replacement of the 5-(1H-pyrrol-1-yl)pyrazole scaffold
}

\section{Claudia Mugnaini, Livia Pasculini, Carlotta Pagli, Antonella Brizzi, Marco Paolino, Beatrice Gianibbi, and Federico Corelli*}

\begin{abstract}
Department of Biotechnology, Chemistry and Pharmacy, University of Siena, 53100 Siena, Italy Email: federico.corelli@unisi.it
\end{abstract}

It is a pleasure to take this opportunity to acknowledge the significant contribution to the chemistry of heterocyclic compounds by Professor Girolamo (Gilmo) Cirrincione and his Palermo group

Received 09-02-2021

Accepted Manuscript 10-03-2021

Published on line $10-09-2021$

\section{Abstract}

Reaction of 3-amino-1H-pyrazole-4-carbonitrile with 2,4-pentanedione yielded 5,7-dimethylpyrazolo[1,5a]pyrimidine-3-carbonitrile, which was easily and efficiently transformed into a small library of amido derivatives. This procedure opens the way to new compounds potentially endowed with interesting biological activities
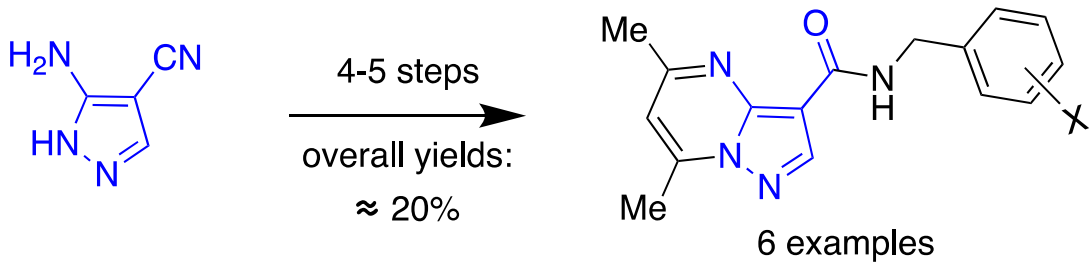

Keywords: Pyrazolo[1,5-a]pyrimidine, bioisosteric replacement, Suzuki coupling, conformational analysis 


\section{Introduction}

Heterocyclic compounds with pyrrole and pyrazole structures have aroused increasing interest over time both for their relevance among natural compounds and for their assessed or potential applications in the pharmaceutical field, as demonstrated by some noteworthy contributions in the recent literature. ${ }^{1-3}$

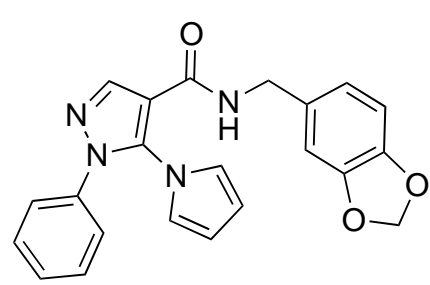

1<smiles>[X]c1cccc(CNC(=O)c2cnn3c(C)cc(C)nc23)c1</smiles>
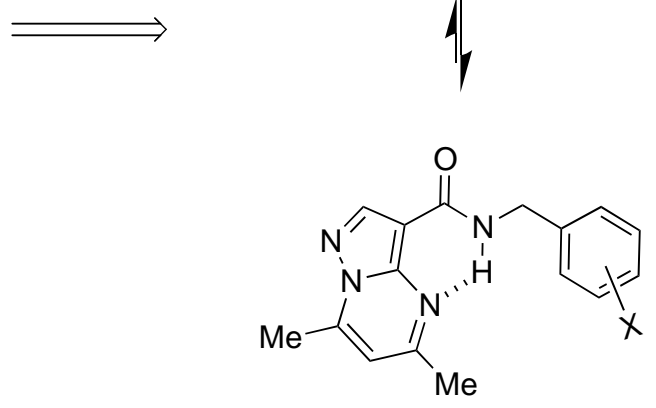

2

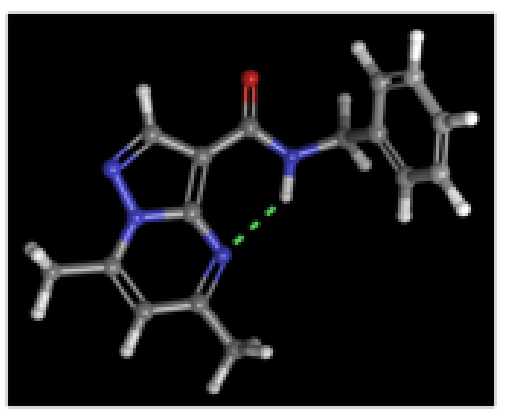

Figure 1. Lead compound 1, general structure of the new compounds 2, and graphical representation of the global minimum conformation of $\mathbf{2}(\mathrm{X}=\mathrm{H})$. The hydrogen bond between the $\mathrm{N} 4$ and the amide $\mathrm{NH}$ is represented by a dashed green line.

Over the years we have thoroughly studied the synthesis and chemical and pharmacological properties of 3-/ 5-pyrrolylpyrazoles. ${ }^{4-7}$ Very recently, through a screening campaign aimed at discovering new antibacterial agents, we identified derivative $\mathbf{1}$ (Figure 1) as a compound endowed with synergistic activity with the known antibiotic colistin. ${ }^{8}$ The significant results obtained prompted us to explore structural modifications of 1 . In particular, we focused on the replacement of the 5-(1H-pyrrol-1-yl)pyrazole scaffold with the condensed pyrazolo[1,5-a]pyrimidine heterocycle, as exemplified by compounds 2 (Figure 1), where position 3 was prioritized as the point of structural divergence.

As shown in previous papers for compounds similar to $1,^{4-6}$ pyrazole and pyrrole rings in compound 1 are far from being coplanar, whereas a pyrazolo[1,5-a]pyrimidine nucleus is expected to ensure the planarity not only of the $10 \pi$-electron heterocycle but also of a wider molecular area as a result of a $\mathrm{H}$-bond interaction between the $\mathrm{NH}$ of the amide group and the nitrogen at the position $4 .{ }^{9}$ This intramolecular hydrogen bond will also help reduce the conformational freedom of the new compounds, thus introducing significant alterations of the three-dimensional structure of the molecule. Furthermore, we wanted to explore the possibility of functionalizing compounds $\mathbf{2}$ with specific amide residues in analogy to what has been recently described by Lu et al. to obtain pyrazolo[1,5-a]pyridine-3-carboxamides with significant antimycobacterial activity. ${ }^{10}$ 


\section{Results and Discussion}

Preliminary molecular modeling studies have referred to the unsubstituted $N$-benzyl-5,7dimethylpyrazolo[1,5-a]pyrimidine-3-carboxamide (Figure 1, $\mathrm{X}=\mathrm{H}$ ). Conformational analysis identified several conformational minima that show a very similar three-dimensional arrangement with the N4---HN distance between 2.20 and $2.23 \AA$, and the NH-N4 angle between 133.9 and 139.6 degrees, comparable to that reported in the literature for similar compounds (i.e., $2.06 \AA$ and 134.7 degrees found for the 6-methyl- $N$ [(1R)-1-[4-(trifluoromethyloxy)phenyl]propyl]pyrazolo[1,5-a]pyrimidine-3-carboxamide co-crystallized to the human PDE2A, entry $5 \times \mathrm{km}$ of the protein data bank), and in agreement with geometric criteria of the existence of the $\mathrm{NH}---\mathrm{N}$ hydrogen bond. ${ }^{11}$

For the synthesis of the bicyclic compounds 2, 2,4-pentanedione was reacted with 3-amino- $1 \mathrm{H}$ pyrazole-4-carbonitrile (3), affording 5,7-dimethylpyrazolo[1,5-a]pyrimidine-3-carbonitrile (4) as a pure crystalline solid in $78 \%$ yield (Scheme 1). The subsequent hydrolysis of the nitrile to carboxylic acid $\mathbf{5}$ was best accomplished by refluxing $\mathbf{4}$ in ethylene glycol with $\mathrm{NaOH}$ overnight. As a whole, the conversion of $\mathbf{3}$ to $\mathbf{5}$ was performed with a satisfactory $55 \%$ overall yield, comparable to that reported by Patnaik et al. ${ }^{12}$

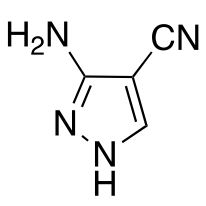

3

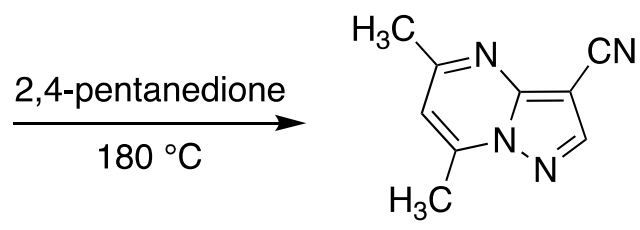

4

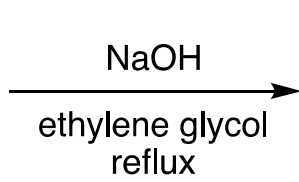

reflux

Scheme 1. Reaction of compound 3 with 2,4-pentanedione and subsequent hydrolysis to carboxylic acid.

With compound $\mathbf{5}$ in our hands, the amides $\mathbf{2 a}$ and $\mathbf{2 b}$ (Scheme 2) were synthesized with yields of $85 \%$ and $65 \%$, respectively, and then subjected to further modification by Suzuki reactions. Thus, reaction with 4 formylphenylboronic acid 2 a provided compound 6, which was directly reduced with $\mathrm{NaBH}_{4}$ to obtain alcohol $\mathbf{2 c}$ in an overall yield of $48 \%$. Compounds $\mathbf{2} \mathbf{d}$-f were synthesized from $\mathbf{2} \mathbf{b}$ by the action of the appropriate boronic acid, namely 3-pyridinylboronic acid, 3-acetamidophenylboronic acid, and 4(trifluoromethoxy)phenylboronic acid, respectively, in $25-67 \%$ yield. All these coupling reactions were conducted under conventional heating, because the use of microwaves as an alternative way gave products in lower yield (for $\mathbf{2} \mathbf{d}$, as an example, $24 \%$ yield instead of $50 \%$ ). 


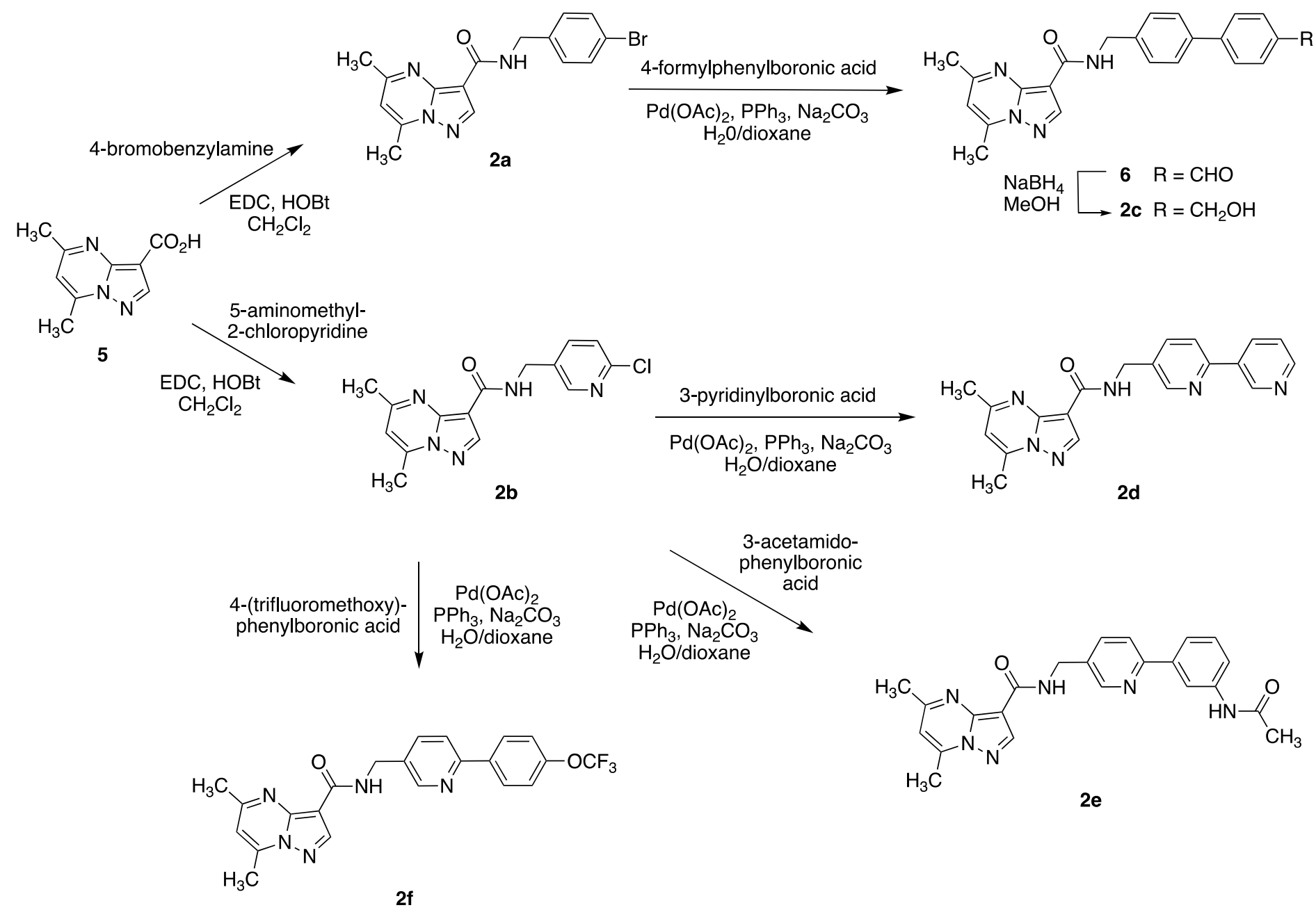

Scheme 2. Synthesis of a small library of 5,7-dimethylpyrazolo[1,5-a]pyrimidine-3-carboxamides.

\section{Conclusions}

Taking compound 1 as a model, an efficient synthetic pathway for functionalization of the pyrazolo[1,5a]pyrimidine scaffold was explored and developed. The results obtained, exemplified by compounds of general structure 2, testify to the possibility of extending this class of heterocyclic compounds into larger libraries in view of their possible biological evaluation.

\section{Experimental Section}

General. Merck silica gel 60 was used for flash chromatography (23-400 mesh). For thin layer chromatography $(T L C)$, silica-coated aluminum plates (Merck Kieselgel $F_{254}$ ) were used. Melting points were determined on a Gallenkamp apparatus and are uncorrected. ${ }^{1} \mathrm{H}$ NMR and ${ }^{13} \mathrm{C}$ NMR were recorded on a Bruker Advance DPX400 or Bruker Avance 600 spectrometers operating at $400 / 100 \mathrm{MHz}$ or $600 / 150 \mathrm{MHz}$, respectively. Chemical shifts ? are in part per million (ppm) relative to TMS as internal standard and coupling constants (J) 
are reported in $\mathrm{Hz}$ (in the attribution of the signals reference is made to the numbering of the bicyclic system reported in Figure 1). Mass spectral (MS) data were obtained using an Agilent 1100 LC/MSD VL system (G1946C) with a $0.4 \mathrm{~mL} / \mathrm{min}$ flow rate using a binary solvent system of 95:5 methanol/water. UV detection was monitored at $254 \mathrm{~nm}$. Elemental analyses were performed with a Perkin-Elmer PE 2400 elemental analyzer and the data for $\mathrm{C}, \mathrm{H}$, and $\mathrm{N}$ are within $0.4 \%$ of the theoretical values. The chemical purity of the target compounds was determined using an Acquity Waters UPLC-MS system under the following conditions: Waters BEH C18 $(2.1 \mathrm{~mm}$ x $50 \mathrm{~mm}, 1.7 \mu \mathrm{m})$ reversed phase column; method: gradient elution, solvent A $(0.1 \%$ formic acid in water), solvent $B(0.1 \%$ formic acid in acetonitrile) 90:10 to 0:100 over 2.9 min, flow rate of 0.5 $\mathrm{mL} / \mathrm{min}$, UV detector, $254 \mathrm{~nm}$. Infrared spectra were recorded on the crystalline powder using an Agilent Cary 630 ATR-FTIR instrument. Microanalyses were performed on a Perkin-Elmer PE 2400 analyzer.

Synthesis of 5,7-dimethylpyrazolo[1,5-a]pyrimidine-3-carbonitrile (4). A mixture of 3-amino-1H-pyrazolo-4carbonitrile (3) $\left(300 \mathrm{mg}, 2.8 \mathrm{mmol}\right.$ ) and 2,4-pentanedione $(4-5 \mathrm{~mL})$ was heated at $180{ }^{\circ} \mathrm{C}$ for $3 \mathrm{~h}$. The solid product that formed on cooling to room temperature was triturated with hexane and recrystallized from EtOH to give 4 as a white solid (373 mg, 78\%). Mp 175-176 ${ }^{\circ} \mathrm{C}$; FT-IR $v_{\max } / \mathrm{cm}^{-1}$ 3119, 3064, 2228 (CN ), 1625 (C=N), 1556, 1423; ${ }^{1} \mathrm{H}$ NMR (600 MHz, $\mathrm{CDCl}_{3}$ ) $\delta 8.31$ (s, 1H, H-2), 6.81 (s, 1H, H-6), 2.79 (s, 3H, Me), 2.66 (s, 3H, Me); ${ }^{13} \mathrm{C}$ NMR $\left(150 \mathrm{MHz}, \mathrm{CDCl}_{3}\right) \delta 163.1,150.2,146.9,146.7,113.2,111.2,81.9,24.8,17.0 ; \mathrm{MS}$ (ESI) $\mathrm{m} / z 173[\mathrm{M}+$ $\mathrm{H}]^{+}$; Found: $\mathrm{C}, 62.59 ; \mathrm{H}, 4.73 ; \mathrm{N}, 32.68 . \mathrm{C}_{9} \mathrm{H}_{8} \mathrm{~N}_{4}$ requires $\mathrm{C}, 62.78 ; \mathrm{H}, 4.68 ; \mathrm{N}, 32.54 \%$.

Synthesis of 5,7-dimethylpyrazolo[1,5-a]pyrimidine-3-carboxylic Acid (5). An aqueous $3 \mathrm{~N}$ solution of $\mathrm{NaOH}$ $(12 \mathrm{~mL}, 36 \mathrm{mmol})$ was added to a suspension of $4(1.2 \mathrm{~g}, 7.0 \mathrm{mmol})$ in ethylene glycol $(20 \mathrm{~mL})$ and the mixture was heated at reflux overnight. After cooling, the reaction mixture was diluted with water, acidified with conc. $\mathrm{HCl}$, and extracted with EtOAc $(7 \times 20 \mathrm{~mL})$. The organic phase was dried over sodium sulfate and evaporated to afford a yellow solid, which was recrystallized from EtOH. The title compound $\mathbf{5}$ was obtained as a yellowish solid (930 mg, 70\%). Mp 178-180 ${ }^{\circ} \mathrm{C}$; FT-IR vmax $/ \mathrm{cm}^{-1}$ 3200-2400 (broad band, COOH), 1625 (C=O), 1553 $(\mathrm{C}=\mathrm{N}) ;{ }^{1} \mathrm{H}$ NMR $\left(400 \mathrm{MHz}\right.$, DMSO-d 6 ) $\delta 8.41(\mathrm{~s}, 1 \mathrm{H}, \mathrm{H}-2), 6.99(\mathrm{~s}, 1 \mathrm{H}, \mathrm{H}-6), 2.62(\mathrm{~s}, 3 \mathrm{H}, \mathrm{Me}), 2.48(\mathrm{~s}, 3 \mathrm{H}, \mathrm{Me}) ;{ }^{13} \mathrm{C}$ NMR $\left(150 \mathrm{MHz}, \mathrm{CDCl}_{3}\right) \delta$ 163.0, 162.6, 147.8 (overlapping of 2 carbon signals), 146.8, 110.6, 101.4, 24.7, 17.0; MS (ESI) $m / z 190[\mathrm{M}-\mathrm{H}]^{-}$; Found: $\mathrm{C}, 56.72 ; \mathrm{H}, 4.69 ; \mathrm{N}, 22.09 . \mathrm{C}_{9} \mathrm{H}_{9} \mathrm{~N}_{3} \mathrm{O}_{2}$ requires $\mathrm{C}, 56.54 ; \mathrm{H}, 4.75 ; \mathrm{N}, 21.98 \%$.

General procedure for the synthesis of amides $\mathbf{2 a}$ and $\mathbf{2 b}$. To a solution of $\mathbf{5}(500 \mathrm{mg}, 2.6 \mathrm{mmol})$ and the appropriate amine $(4.0 \mathrm{mmol})$ in dry $\mathrm{CH}_{2} \mathrm{Cl}_{2}(100 \mathrm{~mL})$ and DMF $(10 \mathrm{~mL}), N$-(3-dimethylaminopropyl)- $N^{\prime}$ ethylcarbodiimide hydrochloride (EDC) $(1.0 \mathrm{~g}, 5.2 \mathrm{mmol})$ and 1-hydroxybenzotriazole (HOBt) (350 mg, 2.6 $\mathrm{mmol}$ ) were added and the mixture was stirred at room temperature for $4 \mathrm{~h}$. Then the reaction mixture was washed with $0.5 \mathrm{~N} \mathrm{HCl}, 10 \% \mathrm{NaHCO}_{3}$ solution, and brine. After drying over sodium sulfate, the organic solution was concentrated in vacuo and the residue was purified as reported below.

$\mathbf{N}$-(4-Bromobenzyl)-5,7-dimethylpyrazolo[1,5-a]pyrimidine-3-carboxamide (2a). Purified by trituration with hexane/Et ${ }_{2} \mathrm{O}$. White solid (650 mg, 70\%); mp 169-172 ${ }^{\circ} \mathrm{C}$; FT-IR vmax/cm ${ }^{-1} 3328(\mathrm{NH}), 1649$ (C=O), 1549, 771; ${ }^{1} \mathrm{H}$ NMR $\left(400 \mathrm{MHz}, \mathrm{CDCl}_{3}\right) \delta 8.59(\mathrm{~s}, 1 \mathrm{H}, \mathrm{H}-2), 8.43$ (br s, 1H, NH), 7.39 (d, J $\left.8.3 \mathrm{~Hz}, 2 \mathrm{H}, \mathrm{Ph}\right), 7.22(\mathrm{~d}, J 8.3 \mathrm{~Hz} 2 \mathrm{H}$, $\mathrm{Ph}), 6.64(\mathrm{~s}, 1 \mathrm{H}, \mathrm{H}-6), 4.61$ (d, J $\left.5.6 \mathrm{~Hz}, 2 \mathrm{H}, \mathrm{CH}_{2}\right), 2.72(\mathrm{~s}, 3 \mathrm{H}, \mathrm{Me}), 2.53$ (s, 3H, Me); ${ }^{13} \mathrm{C} \mathrm{NMR}\left(100 \mathrm{MHz} \mathrm{CDCl}_{3}\right) \delta$ $162.8,161.1,147.0,146.1,146.0,138.3,131.6$ (2 equivalent carbons), 129.2 (2 equivalent carbons), 120.9, 109.7, 104.6, 42.3, 24.8, 17.0; MS (ESI) m/z 359 (100\%), 361 (98\%) [M + H] ; Found: C, 53.68; H, 4.17; N, 15.47. $\mathrm{C}_{16} \mathrm{H}_{15} \mathrm{BrN}_{4} \mathrm{O}$ requires $\mathrm{C}, 53.50 ; \mathrm{H}, 4.21 ; \mathrm{N}, 15.60 \%$.

$\mathbf{N}$-[(6-Chloropyridin-3-yl)methyl]-5,7-dimethylpyrazolo[1,5-a]pyrimidine-3-carboxamide (2b). Purified by flash chromatography (silica, EtOAc/hexane 2:1 to 4:1). White crystals (534 mg, 65\%); mp 157-160 ${ }^{\circ} \mathrm{C}$; FT-IR vmax/cm ${ }^{-1} 3324(\mathrm{NH}), 2918,1650(\mathrm{C}=0), 1551,1458,773 ;{ }^{1} \mathrm{H} \mathrm{NMR}\left(400 \mathrm{MHz}, \mathrm{CDCl}_{3}\right) \delta 8.53(\mathrm{~s}, 1 \mathrm{H}, \mathrm{H}-2), 8.43$ 
(br s, 1H, NH), 8.34 (s, 1H, Py), 7.68 (d, J 8.2 Hz, 1H, Py), 7.21 (d, J 8.2 Hz, 1H, Py), 6.64 (s, 1H, H-6), 4.63 (d, J 5.9 $\mathrm{Hz}, 2 \mathrm{H}, \mathrm{CH}_{2}$ ), 2.71 (s, 3H, Me), 2.53 (s, 3H, Me). ${ }^{13} \mathrm{C} \mathrm{NMR} \mathrm{(100} \mathrm{MHz,} \mathrm{CDCl} 3$ ) $\delta 162.8,161.3,150.1,148.8,147.0$, 146.0, 145.9, 138.4, 134.0, 124.1, 109.8, 104.2, 39.6, 24.7, 17.0; MS (ESI) $m / z 316$ (100\%), 318 (32\%) [M + H] ; Found: $\mathrm{C}, 56.90 ; \mathrm{H}, 4.40 ; \mathrm{N}, 21.99$. $\mathrm{C}_{15} \mathrm{H}_{14} \mathrm{ClN}_{5} \mathrm{O}$ requires $\mathrm{C}, 57.06 ; \mathrm{H}, 4.47 ; \mathrm{N}, 22.18 \%$.

General procedure for the synthesis of compounds $\mathbf{2 d}, \mathbf{2 e}, \mathbf{2 f}$. In a $25 \mathrm{~mL}$ round bottom flask $\mathbf{2 a}$ or $\mathbf{2 b}(0.63$ $\mathrm{mmol}$ ) was dissolved in 1,4-dioxane (3-4 mL) under nitrogen. Then the appropriate arylboronic acid (1.9 mmol, $3 \mathrm{eq}$ ), $\mathrm{PPh}_{3}\left(50 \mathrm{mg}, 0.19 \mathrm{mmol}, 3 \mathrm{eq}\right.$ ), $\mathrm{Pd}(\mathrm{OAc})_{2}(14 \mathrm{mg}, 0.063 \mathrm{mmol}, 0.1 \mathrm{eq}$ ) were added successively, followed by addition of EtOH $(2 \mathrm{~mL})$ and $1 \mathrm{M} \mathrm{Na}_{2} \mathrm{CO}_{3}(1.27 \mathrm{~mL}, 1.27 \mathrm{mmol}, 2 \mathrm{eq})$. The mixture was refluxed for $6 \mathrm{~h}$, then cooled to room temperature and filtered through celite. The yellow solution was dried over sodium sulfate and concentrated in vacuo to leave a solid residue which was purified as described below.

$\mathbf{N}$-[(2,3'-Bipyridin)-5-ylmethyl]-5,7-dimethylpyrazolo[1,5-a]pyrimidine-3-carboxamide (2d). Prepared from $\mathbf{2 b}$ and 3-pyridinylboronic acid. Purified by flash chromatography (silica, EtOAc to EtOAc/MeOH 9:1). White solid (152 mg, 67\% yield); mp 184-186 ${ }^{\circ} \mathrm{C}$; FT-IR vmax/cm ${ }^{-1} 3324(\mathrm{NH}), 2922,1651$ (C=O), 1557, 1374, 707; ${ }^{1} \mathrm{H}$ NMR (400 MHz, CDCl 3 ) $\delta 9.20(\mathrm{~s}, 1 \mathrm{H}, \mathrm{Py}), 8.72(\mathrm{~s}, 1 \mathrm{H}, \mathrm{H}-2), 8.61-8.52(\mathrm{~m}, 4 \mathrm{H}, \mathrm{Py}$ and $\mathrm{NH}), 7.87(\mathrm{~d}, J 8.1 \mathrm{~Hz}, 1 \mathrm{H}$, Py), 7.71 (d, J 8.1 Hz, 1H, Py), 7.59-7.52 (m, 1H, Py), 6.66 (s, 1H, H-6), 4.74 (d, J 5.7 Hz, 2H, CH $), 2.73$ (s, 3H, $\mathrm{Me}), 2.56(\mathrm{~s}, 3 \mathrm{H}, \mathrm{Me}) ;{ }^{13} \mathrm{C}$ NMR $\left(100 \mathrm{MHz}\right.$, DMSO- $\left.d_{6}\right) \delta 162.2,162.0,152.9,150.2,149.5,148.1,147.5,145.8$, $145.5,136.8,135.4,134.4,134.2,124.2,120.8,110.6,104.4,24.8,16.9$, one more signal overlapped with solvent; MS (ESI) $m / z 359[\mathrm{M}+\mathrm{H}]^{+}$; Found: $\mathrm{C}, 67.22 ; \mathrm{H}, 5.00 ; \mathrm{N}, 23.36 . \mathrm{C}_{20} \mathrm{H}_{18} \mathrm{~N}_{6} \mathrm{O}$ requires $\mathrm{C}, 67.02 ; \mathrm{H}, 5.06 ; \mathrm{N}$, $23.45 \%$.

N-[[6-(3-Acetamidophenyl)pyridin-3-yl]methyl]-5,7-dimethylpyrazolo[1,5-a]pyrimidine-3-carboxamide (2e). Prepared from $\mathbf{2 b}$ and 3-acetamidophenylboronic acid. Purified by flash chromatography (silica, EtOAc to EtOAc/MeOH 9:1). White solid (135 mg, 34\% yield); mp 232-233 ${ }^{\circ} \mathrm{C}$; FT-IR vmax/cm ${ }^{-1} 3308(\mathrm{NH}), 1680$ (C=O), 1636 (C=O), 1549, 1471, 707; ${ }^{1} \mathrm{H}$ NMR (400 MHz, DMSO-d 6 ) $\delta 9.97$ (s, 1H, AcNH), 8.59 (s, 1H, Py), 8.50 (t, J 5.8 $\left.\mathrm{Hz}, 1 \mathrm{H}, \mathrm{NH}-\mathrm{CH}_{2}\right), 8.45$ (s, 1H, H-2), 8.19 (s, 1H, Py), 7.77 (s, 2H, Ph), 7.67-7.57 (m, 2H, Ph and Py), 7.30 (t, J 7.9 $\mathrm{Hz}, 1 \mathrm{H}, \mathrm{Ph}$ ), 7.02 (s, 1H, H-6), 4.59 (d, J $5.9 \mathrm{~Hz}, 2 \mathrm{H}, \mathrm{CH}_{2}$ ), 2.65 (s, 3H, Me), 2.52 (s, 3H, Me), 1.99 (s, 3H, MeCO); ${ }^{13} \mathrm{C}$ NMR $\left(100 \mathrm{MHz}\right.$, DMSO-d $\left.d_{6}\right) \delta 168.9,162.2,162.0,155.0,149.1,147.4,145.8,145.5,140.3,139.4,136.6$, 134.7, 129.5, 121.5, 120.3, 120.0, 117.5, 110.5, 104.4, 40.0 (overlapped with solvent), 24.8, 24.5, 16.9; MS (ESI) $\mathrm{m} / \mathrm{z} 415[\mathrm{M}+\mathrm{H}]^{+}$; Found: $\mathrm{C}, 66.80 ; \mathrm{H}, 5.29 ; \mathrm{N}, 20.15 . \mathrm{C}_{23} \mathrm{H}_{22} \mathrm{~N}_{6} \mathrm{O}_{2}$ requires $\mathrm{C}, 66.65 ; \mathrm{H}, 5.35 ; \mathrm{N}, 20.28 \%$.

\section{5,7-Dimethyl-N-[[6-[4-(trifluoromethoxy)phenyl]pyridin-3-yl]methyl]pyrazolo[1,5-a]pyrimidine-3-}

carboxamide (2f). Prepared from $\mathbf{2 b}$ and 4-(trifluoromethoxy)phenylboronic acid. Purified by flash chromatography (silica, EtOAc/hexane 4:1). Colorless solid (136 mg, 54\% yield); mp 206-207 ${ }^{\circ} \mathrm{C}$; FT-IR vmax/cm ${ }^{-1} 3309(\mathrm{NH}), 1653(\mathrm{C}=0), 1545,1474,1265-1159(\mathrm{C}-\mathrm{F}) ;{ }^{1} \mathrm{H} \mathrm{NMR}\left(400 \mathrm{MHz}, \mathrm{CDCl}_{3}\right) \delta 8.69(\mathrm{~s}, 1 \mathrm{H}$, $\mathrm{Ph})$, 8. 57 (s, 1H, H-2), $8.51(\mathrm{t}, J 5.5 \mathrm{~Hz}, 1 \mathrm{H}, \mathrm{NH}), 7.98$ (d, J $8.7 \mathrm{~Hz}, 2 \mathrm{H}, \mathrm{Ph}), 7.90(\mathrm{~d}, J 8.0 \mathrm{~Hz}, 1 \mathrm{H}, \mathrm{Py}), 7.66$ (d, J 8.2 $\mathrm{Hz}, 1 \mathrm{H}, \mathrm{Py}), 7.25$ (d, J $8.4 \mathrm{~Hz}, 2 \mathrm{H}, \mathrm{Ph}), 6.65$ (s, 1H, H-6), 4.73 (d, J $\left.6.0 \mathrm{~Hz}, 2 \mathrm{H}, \mathrm{CH}_{2}\right), 2.73(\mathrm{~s}, 3 \mathrm{H}, \mathrm{Me}), 2.55(\mathrm{~s}, 3 \mathrm{H}$, $\mathrm{Me}) ;{ }^{13} \mathrm{C}$ NMR $\left(100 \mathrm{MHz}_{\mathrm{CDCl}}\right.$ ) $\delta 162.8,161.2,154.5,149.9,148.5,147.0,146.0,145.9,137.2,136.9,134.0$, 128.4 (overlapped signals for 3 carbons), 121.7 (signal for $\mathrm{C}-\mathrm{F}$ coupling), 121.0 (signal for $\mathrm{C}-\mathrm{F}$ coupling and 1 more carbon signal), 120.4 (signal for $\mathrm{C}-\mathrm{F}$ coupling and 1 more carbon signal), 119.2 (signal for $\mathrm{C}-\mathrm{F}$ coupling), 109.7, 104.4, 40.1, 24.7, 16.9; MS (ESI) $\mathrm{m} / \mathrm{z} 442[\mathrm{M}+\mathrm{H}]^{+}$; Found: $\mathrm{C}, 60.08 ; \mathrm{H}, 4.18$; N, 15.70. $\mathrm{C}_{22} \mathrm{H}_{18} \mathrm{~F}_{3} \mathrm{~N}_{5} \mathrm{O}_{2}$ requires $\mathrm{C}, 59.86 ; \mathrm{H}, 4.11 ; \mathrm{N}, 15.87 \%$.

Synthesis of $\mathbf{N}$-[[4'-(hydroxymethyl)-(1,1'-biphenyl)-4-yl]methyl]-5,7-dimethylpyrazolo[1,5-a]pyrimidine-3carboxamide (2c). Reaction of 2 a $(200 \mathrm{mg}, 0.56 \mathrm{mmol})$ with 3 -formylboronic acid according to the general procedure reported above afforded $N$-[[4'-formyl-(1,1'-biphenyl)-4-yl]methyl]-5,7-dimethylpyrazolo[1,5a]pyrimidine-3-carboxamide (6) (135 mg, 63\%) after purification of the crude by flash chromatography (sílica, 
EtOAc/hexane 2:1). The aldehyde $6(100 \mathrm{mg}, 0.26 \mathrm{mmol})$ was dissolved in $\mathrm{MeOH}(20 \mathrm{~mL})$ and treated with $\mathrm{NaBH}_{4}(10 \mathrm{mg}, 0.26 \mathrm{mmol})$ at room temperature for $4 \mathrm{~h}$. The reaction mixture was concentrated and diluted with water and EtOAc. The organic layer was separated, dried over sodium sulfate, filtered, and evaporated to dryness. The residue was recrystallized from $\mathrm{MeOH} /$ EtOAc to give $\mathbf{2 c}(77 \mathrm{mg}, 77 \%, 48 \%$ overall yield from $\mathbf{2 a}$ ). White solid; mp 223-225 ${ }^{\circ} \mathrm{C}$; FT-IR vmax/cm ${ }^{-1} 3323(\mathrm{NH}), 1647$ (C=O), 1556, 1425, 1375; ${ }^{1} \mathrm{H}$ NMR (400 MHz, DMSO-d $\left.d_{6}\right) \delta 8.45$ (superimposed signals, $2 \mathrm{H}, \mathrm{H}-2$ and $\mathrm{NH}$ ), 7.62-7.48 (m, 4H, Ph), $7.36(\mathrm{~d}, J 7.8 \mathrm{~Hz}, 2 \mathrm{H}, \mathrm{Ph}), 7.31$ (d, J $7.8 \mathrm{~Hz}, 2 \mathrm{H}, \mathrm{Ph}), 7.02$ (s, 1H, H-6), 5.11 (br s, $1 \mathrm{H}, \mathrm{OH}), 4.57$ (d, J $\left.5.6 \mathrm{~Hz}, 2 \mathrm{H}, \mathrm{CH}_{2}-\mathrm{NH}\right), 4.45(\mathrm{~s}, 2 \mathrm{H}, \mathrm{CH}-\mathrm{OH})$, 2.66 (s, 3H, Me), 2.52 (s, 3H, Me); ${ }^{13} \mathrm{C}$ NMR (100 MHz, DMSO-d 6 ) $\delta$ 162.0, 161.9, 147.5, 145.8, 145.5, 142.2, $139.2,139.1,138.8,128.2$ (2 equivalent carbons), 127.5 (2 equivalent carbons), 127.0 (2 equivalent carbons), 126.7 (2 equivalent carbons), 110.5, 104.5, 63.1, 42.1, 24.8, 16.9; MS (ESI) $\mathrm{m} / \mathrm{z} 387$ [M + H] ; Found: C, 71.68; $\mathrm{H}, 5.66 ; \mathrm{N}, 14.69$. $\mathrm{C}_{23} \mathrm{H}_{22} \mathrm{~N}_{4} \mathrm{O}_{2}$ requires $\mathrm{C}, 71.48 ; \mathrm{H}, 5.74 ; \mathrm{N}, 14.50 \%$.

Molecular modeling. The LigPrep routine of Maestro was applied to convert 1D SMILES notation into a low energy 3D structure of the studied compound. ${ }^{13}$ Next, a systematic torsional sampling of the rotatable bonds (namely, a systematic pseudo-Monte Carlo conformational search within the ConfGen routine ${ }^{14}$ ) was performed, using the OPLS3e force field and water, chloroform, or DMSO as the solvent. Conformations within $25 \mathrm{kcal} / \mathrm{mol}$ above the global minimum were minimized with 1000 iterations of the Polak-Ribiere conjugate gradient algorithm.

\section{Acknowledgements}

Authors acknowledge the partial support by MIUR Progetto Dipartimenti di Eccellenza 2018-2022, grant n. L. 232/2016.

\section{Supplementary Material}

Copies of the ${ }^{13} \mathrm{C}$ NMR spectra and HPLC traces of compounds 2a-f are given in the Supplementary Material file associated with this manuscript.

\section{References}

1. Diana, P.; Cirrincione, G. Biosynthesis of Heterocycles: from isolation to gene cluster; John Wiley \& Sons, Inc.: Hoboken, NJ, 2015.

2. Li Petri, G.; Spanò, V.; Spatola, R.; Holl, R.; Raimondi, M. V.; Barraja, P.; Montalbano, A. Eur. J. Med. Chem. 2020, 208, 112783. doi.org/10.1016/j.ejmech.2020.112783

3. Karrouchi, K.; Radi, S.; Ramli, Y.; Taoufik, J.; Mabkhot, Y. N.; Al-aizari, F. A.; Ansar, M. Molecules 2018, 23, 134-220.

doi.org/10.3390/molecules23010134

4. Silvestri, R.; Cascio, M. G.; La Regina, G.; Piscitelli, F.; Lavecchia, A.; Brizzi, A.; Pasquini, S.; Botta, M.; Novellino, E.; Di Marzo, V.; Corelli, F. J. Med. Chem. 2008, 51, 1560-1576. 


\section{doi.org/10.1021/jm070566z}

5. Silvestri, R.; Ligresti, A.; La Regina, G.; Piscitelli, F.; Gatti, V.; Brizzi, A.; Pasquini, S.; Lavecchia, A.; Allarà, M.; Fantini, N.; Carai, M. A. M.; Novellino, E.; Colombo, G.; Di Marzo, V.; Corelli, F. Bioorg. Med. Chem. 2009, $17,5549-5564$.

doi:10.1016/j.bmc.2009.06.027

6. Silvestri, R.; Ligresti, A.; La Regina, G.; Piscitelli, F.; Gatti, V.; Lavecchia, A.; Brizzi, A.; Pasquini, S.; Allarà, M.; Fantini, N.; Carai, M. A. M.; Bigogno, C.; Rozio, M. G.; Sinisi, R.; Novellino, E.; Colombo, G.; Di Marzo, V.; Dondio, G.; Corelli, F. Eur. J. Med. Chem. 2010, 45, 5878-5886.

doi.org/10.1016/i.ejmech.2010.09.053

7. Piscitelli, F.; Ligresti, A.; La Regina, G.; Gatti, V.; Brizzi, A.; Pasquini, S.; Allarà, M.; Carai, M. A. M.; Novellino, E.; Colombo, G.; Di Marzo, V.; Corelli, F.; Silvestri, R. Eur. J. Med. Chem. 2011, 46, 5641-5653. doi.org/10.1016/i.ejmech.2011.09.037

8. Mugnaini, C.; Sannio, F.; Brizzi, A.; Del Prete, R.; Simone, T.; Ferraro, T.; De Luca, F.; Corelli, F.; Docquier, J.D. ACS Med. Chem. Lett. 2020, 11, 899-905. doi.org/10.1021/acsmedchemlett.9b00674

9. Mikami, S.; Sasaki, S.; Asano, Y.; Ujikawa, O.; Fukumoto, S.; Nakashima, K.; Oki, H.; Kamiguchi, N.; Imada, H.; Iwashita, H.; Taniguchi, T. J. Med. Chem. 2017, 60, 7658-7676.

doi.org/10.1021/acs.jmedchem.7b00709

10. Lu, X.; Tang, J.; Cui, S.; Wan, B.; Franzblau, S. G.; Zhang, T.; Zhang, X.; Ding, K. Eur. J. Med. Chem. 2017, 125, 41-48.

doi.org/10.1016/i.ejmech.2016.09.030

11. Grabowski, S. J. Understanding Hydrogen Bonds: Theoretical and Experimental Views. Theoretical and Computational Chemistry Series No. 19. Hydrogen Bond - Definitions, criteria of existence and various types. Chapter 1, pp. 1-40, Royal Society of Chemistry, 2021.

12. Patnaik, S.; Zheng, W.; Choi, J. H.; Motabar, O.; Southall, N.; Westbroek, W.; Lea, W. A.; Velayati, A.; Goldin, E.; Sidransky, E.; Leister, W.; Marugan, J. J. J. Med. Chem. 2012, 55, 5734-5748. doi.org/10.1021/jm300063b

13. Maestro version 11.9, release 2019-1, Schrodinger, LLC, New York, NY, 2019.

14. Watts, K. S.; Dalal, P.; Murphy, R. B.; Sherman, W.; Friesner, R. A.; Shelley, J. C. J. Chem. Inf. Model. 2010, 50, 534-546.

doi.org/10.1021/ci100015j 